\section{Spontaneous sublingual and intramural small-bowel hematoma in a patient on oral anticoagulation}

\author{
Mohamed Moftah, ${ }^{1}$ Ronan Cahill, ${ }^{1}$ \\ Sean Johnston ${ }^{2}$ \\ 1Department of Colorectal Surgery, \\ Beaumont Hospital, Dublin; ${ }^{2}$ Department \\ of Surgery Tallamore General Hospital, \\ Co. Offaly, Ireland
}

\section{Abstract}

Spontaneous sublingual hematoma and intramural small bowel hematoma are rare and serious complications of anticoagulant therapy. Though previously reported individually, there has been no previous report of the same two complications occurring in a single patient. A 71-year-old Caucasian man, who was on warfarin for atrial fibrillation, presented with difficulty in swallowing due to a sublingual hematoma. He was observed in our intensive care unit, his warfarin was held and he recovered with conservative management. He represented two months later with a two day history of abdominal pain and distension. An abdominopelvic computed tomography (CT) scan now showed small bowel obstruction due to intramural small bowel hematoma and haemorrhagic ascites. Again, this was treated expectantly with a good outcome. In conclusion, life threatening haemorrhagic complications of oral anticoagulant therapy can recur. Conservative treatment is successful in most cases, but an accurate diagnosis is mandatory to avoid unnecessary surgery. CT scan is the investigation of choice for the diagnosis of suspected haemorrhagic complications of over coagulation.

\section{Introduction}

Non-traumatic spontaneous haematomas due to oral anticoagulation are rare complications that can occur anywhere in the gastrointestinal tract. Though most of these cases have a typical pattern of presentation, there is no consensus on their management. Their rarity can pose a diagnostic challenge and an invasive intervention can be avoided if an accurate diagnosis is made. Future understanding of the aetiopathogenesis of these complications would facilitate establishing guidelines in their management.

\section{Case Report}

A 71 year-old Caucasian male presented to the emergency department with an eight hour history of sublingual swelling and drooling saliva and neck pain two days after commencing oral amoxicillin (500 mg qds) for sore throat. There was no history of recent trauma and did not use tobacco or alcohol. One year ago, he was diagnosed with atrial fibrillation and warfarin was started in a daily dose of 3 mg. Other once daily medications were Atorvastatin $20 \mathrm{mg}$, lanoxin PG 2 tab and Bisoprolol $2.5 \mathrm{mg}$. His only previous surgery was a laparoscopic cholecystectomy.

On physical examination he was in no acute distress and his vital signs were normal except for tachycardia (101 bpm). Oxygen saturation was $98 \%$ on room air. Oral examination revealed a large sublingual haematoma while the sub-mental triangle was swollen and tender to palpation.

Initial laboratory data revealed international normalized ratio (INR) of 6.6 and prothrombin time (PT) of $75.5 \mathrm{~s}$ (control: 11.5-15.0). Other haematological parameters were normal (including haemoglobin and platelet count). He was admitted to intensive care, kept nil by mouth and given intravenous fluids. Warfarin was held and he was treated with intravenous vitamin $\mathrm{K}, 5 \mathrm{mg}$ and hydrocortisone, $100 \mathrm{mg}$. Our otolaryngology service advised close monitoring unless airway compromise progressed. Six hours later, INR was 2.8 and PT 26.2 seconds (11.5-15.0). He remained stable and neither intubation nor surgical airway was required. Twenty-four hours later, his INR was 1.4 and PT was 19.5 seconds. His swallowing improved and he began soft diet. After two days, he was discharged to the ward with INR of 1.7 and haemoglobin of $16.4 \mathrm{~g} / \mathrm{dL}$. During his hospital stay he reported having chronic diarrhoea for one year and was investigated with thyroid function tests, celiac screen and small bowel follow (all normal). He was discharged home well after 8 days being advised to stop warfarin. Review four weeks later showed his sublingual hematoma had resolved completely. He was still complaining of two to three episodes of diarrhoea each day.

Two months later, he represented with a two day history of central colicky abdominal pain, nausea and constipation. There was no history of abdominal trauma. On physical examination, he was in mild distress but vital signs were stable. Abdominal examination revealed generalized mild tenderness mainly in the left upper quadrant with hypoactive bowel sounds. Digital rectal examination was normal and faecal occult blood was negative. Chest examination revealed decreased air entry and crepitation at base of left lung. Haemoglobin was $16.6 \mathrm{~g} / \mathrm{dL}$, hematocrit $0.477 \mathrm{~L} / \mathrm{L}$, white cell count $14.8 \times 10^{9}$,
Correspondence: Mohamed Moftah, Department of Colorectal Surgery, Beaumont Hospital, 305 Carrington, Northwood, Santry, Dublin 9, Ireland. E-mail:mmoftah@rcsi.ie

Key words: spontaneous small bowel haematoma, warfarin toxicity, small bowel obstruction.

Conflict of interests: the authors report no potential conflict of interests.

Received for publication: 30 September 2011. Accepted for publication: 14 May 2012.

This work is licensed under a Creative Commons Attribution NonCommercial 3.0 License (CC BYNC 3.0).

(C) Copyright M. Moftah et al., 2012

Licensee PAGEPress, Italy

Gastroenterology Insights 2012; 4:e17

doi:10.4081/gi.2012.e17

and platelet count of 217 . Coagulation profile was significant for an INR of 11.2, and PT of 118 seconds (11.5-15.0). Electrolytes were normal. Plain film abdomen revealed a dilated loop of small bowel in the left upper quadrant with thickening of bowel wall (Figure 1). An ultrasound scan of abdomen showed dilated loops of small bowel and free fluid in pelvis.

A tentative diagnosis of small bowel obstruction was made and he was admitted. He was kept nil by mouth and started on intravenous fluids. Intravenous vitamin $\mathrm{K}, 2 \mathrm{mg}$ was given and nasogastric tube inserted. Ten hours later, INR was 3 and PT was 38.1 however his haemoglobin dropped to $13.9 \mathrm{~g} / \mathrm{dL}$ and haematocrit to 0.40 . A further dose of IV vitamin K, 5 mg was given and INR dropped to 1.8 and PT to 24.7 after 6 hours. Computed tomography (CT) of abdomen showed a distended and fluid filled loop of proximal jejunum with inflammatory stranding in the adjacent mesentery. There was diffuse mucosal thickening and luminal narrowing with moderate amount of free fluid in the pelvis and around the spleen (Figures 2 and 3). These findings were consistent with intramural small bowel haematoma and haemorrhagic ascites.

Over the following days, his condition improved and became pain free. He had a few episodes of melena which settled spontaneously. He was commenced on oral diet, cautioned about further use of warfarin and discharged home 5 days later. Upon discharge, his INR was 1.7, and PT was 22.7, haemoglobin 12.9 , haematocrit 0.368 . Barium enema performed during this admission was normal. A repeat CT after 3 weeks showed complete resolution of bowel haematoma and haemorrhagic ascites (Figure 3).

Two months later, he was assessed at med- 
ical clinic and commenced on $75 \mathrm{mg}$ aspirin, once daily. A year later, he remained well, continued on aspirin and diarrhoea settled completely.

\section{Discussion}

Oral anticoagulants are commonly prescribed for patients at risk for thromboembolism. In the United States alone, it has been estimated that 2.3 million patients currently have atrial fibrillation, approximately $40 \%$ of whom are receiving anticoagulants. ${ }^{1}$ Haemorrhagic complications of oral anticoagulation can occur in any organ, ${ }^{2}$ but the genitourinary, gastrointestinal, skin and nose are most commonly affected. Sublingual hematoma and intramural small bowel haematoma are both rare, but life-threatening complications. Less than twenty cases of sublingual haematoma and more than a hundred cases of intramural small haematoma have been reported in the literature. We suspected that taking warfarin inadvertently is the most likely cause of INR prolongation in the second presentation of our patient. Plasma warfarin level would have provided a clear cut evidence of warfarin overdose, if it was considered at that time. Warfarin can cause persistent diarrhoea and we believe it is the cause in our patient, as all investigations for diarrhoea were normal and his diarrhoea completely subsided after permanently stopping the warfarin. Moreover, diarrhoea may result in malabsorption of vita$\min \mathrm{K}$, which can lead to INR elevations. ${ }^{3}$

What determines the location of haematoma in the gastrointestinal tract is unclear. In most of the cases that have been reported, there was no history of trauma or medications that potentiated the hypoprothrombinemic effects of warfarin or interfered with coagulation. Pathologic examination of resected bowel specimens show only submucosal and intramural haemorrhage. 4 The gradual onset of symptoms could reflect the slow bleeding caused by small vessels under the mucosa. In cases of lingual haematoma, including our patient, sore throat or cough for few days prior to presentation was a common finding. ${ }^{5}$ It is not known if these symptoms are secondary to irritation caused by slowly growing hematoma or actually are precipitating factors for the development of haematoma.

The clinical presentation depends on the location and extent of the developing haematoma. In the sublingual region, haematoma can compromise airways and those affecting bowel could cause bowel obstruction. Diagnosis can be clinically evident, as in case of sublingual haematoma, or occult requiring a high index of suspicion and further investigations, as in case of small bowel hematoma. Acute abdominal pain with symptoms of bowel obstruction in a patient on anticoagulation therapy should raise suspicion of spontaneous intramural small bowel haematoma. Plain film radiography may show dilatation and thickening of the intestinal wall, but these are not specific findings and present in only a small percentage of cases. ${ }^{6}$ Gastrointestinal studies with barium were used in the past for diagnosis but will only show abnormality in lumen or mucosa of the bowel. Though ultrasound scan can show intramural bowel hematoma, its sensitivity is much less than CT, especially in obese patients. ${ }^{7}$ The triad of warfarin toxicty, circumferential bowel wall thickening and intestinal obstruction, described by Abbas et al., as seen in our patient's CT, is

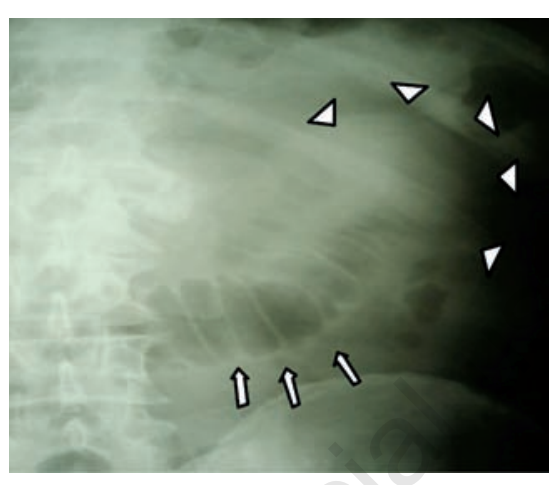

Figure 1. Dilated loop of small bowel (arrows) and thickenend bowel wall (arrow heads) on plain film abdomen. pathognomonic for intramural small bowel haematoma. ${ }^{8}$

There is little consensus regarding the conservative management of these rare complications. Sorbello et al. reviewed 28 case reports of spontaneous small bowel haematoma over 25 years period and observed that surgical exploration, which was previously used as a diagnostic method, has now become primarily a treatment method reserved for cases with complications like suspected ischemia with or without perforation and peritonitis. Conservative therapy is now favoured and a logical approach would be: ${ }^{9}$ hold anticoagulant, reverse hypercoagulation, and treat the haematoma. How quickly

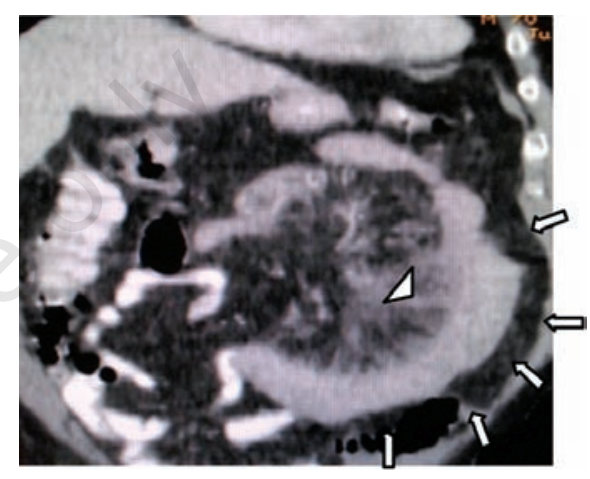

Figure 2. Computed tomography shows dilated and thickened jejunum (arrows) and mesenteric stranding (arrow heads).

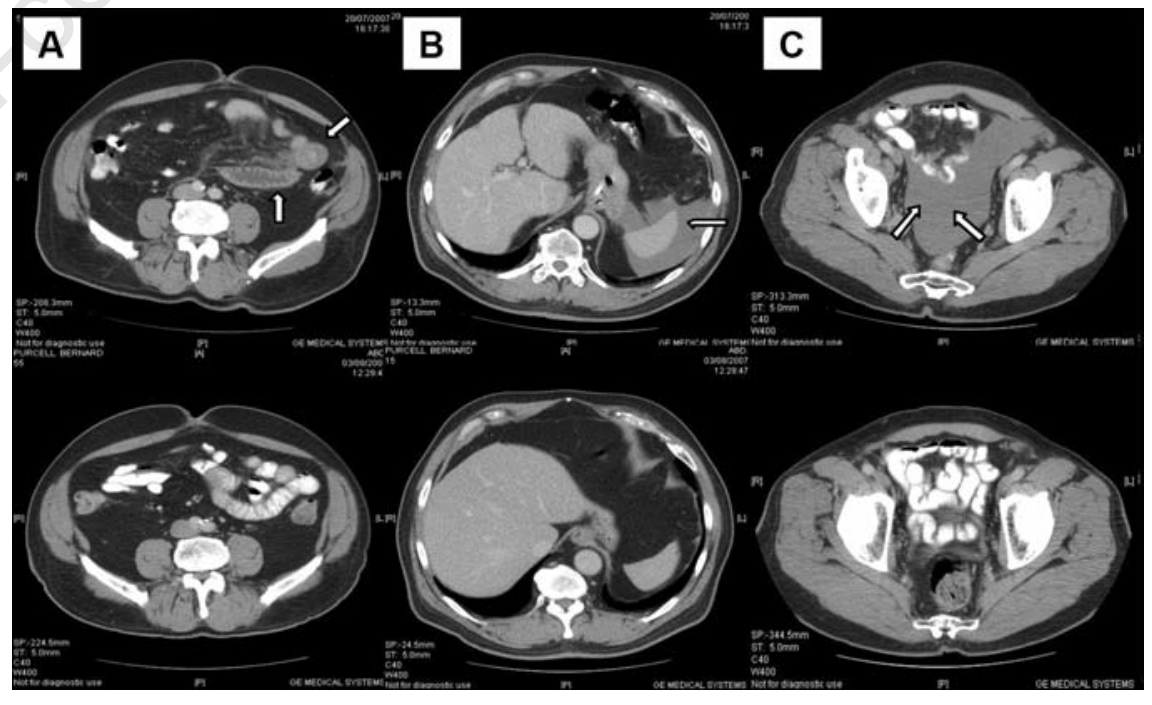

Figure 3. Computed tomography showing cross-sectional and longitudinal images at both presentation (top row) and then three weeks later (bottom row). A) Shows thick wall jejunum (arrows); B) shows haemorrhagic ascites around spleen (arrow) and C) in pelvis (arrows). Underneath each image is the corresponding axial cut from a repeat computed tomography abdomen and pelvis performed three weeks afterwards showing complete resolution of the abnormality. 
should we reverse coagulation is debatable. Successful rapid reversal with prothrombin complex concentrate was obtained in one case report of sublingual haematoma although this risks thrombogenesis. ${ }^{10}$ The insidious development of haematoma in most of the reported cases would suggest that reversal need not be achieved so rapidly. The Seventh ACCP Conference on Antithrombotic and Thrombolytic Therapy recommended administering Vitamin K1 (10 mg by slow IV infusion) supplemented with Fresh Plasma, Prothrombin Complex Concentrate, or Recombinant Factor VIIa, depending on the urgency of the situation (grade 1C). ${ }^{11}$ As in our patient, reversal of warfarin was obtained within few hours of administering vitamin $\mathrm{K}$ intravenously with fewer chances of thrombotic events. Most authors think it is safe to resume anticoagulant therapy in patients as long as it is administered within the therapeutic range. Aspirin is an alternative, but it is not as effective thromboprophylactic agent as warfarin. ${ }^{12}$

\section{Conclusions}

In conclusion, oral anticoagulation can cause serious haemorrhagic complications in the gastrointestinal tract. Some of these com- plications can be clinically evident, as in case of sublingual haematoma. Others need high index of suspicion and radiographic evaluation to avoid unnecessary surgery. Reversal of coagulation, close monitoring and observation constitute a reasonable management option in the majority of cases.

\section{References}

1. Stafford RS, Singer DE. Recent national patterns of warfarin use in atrial fibrillation. Circulation 1998;97:1231-3.

2. Sinert R, Scalea T. Retropharyngeal and bowl hamatomas in an anticoagulated patient. Acad Emerg Med 1994;1:67-72.

3. Smith JK, Aljazairi A, Fuller SH. INR elevation associated with diarrhea in a patient receiving warfarin. Ann Pharmacother 1999;33:301-4 .

4. Polat C, Dervisoglu A, Guven H, et al. Anticoagulant-induced intramural intestinal hematoma. Am J Emerg Med 2003;21: 208-11.

5. Cohen AF, Warman SP. Upper airway obstruction secondary to warfarin-induced sublingual hematoma. Arch Otolaryngol Head Neck Surg 1989;115:718-20.

6. Sears AD, Hawkins J, Kilgore BB, Miller
JE. Plain roentgenographic findings in drug induced intramural hematoma of the small bowel. Am J Roentgenol Radium Ther Nucl Med 1964;91:808-13.

7. Rauh P, Uhle C, Ensberg D, et al. sonographic characteristics of intramural bowel hematoma. J Clin Ultrasound 2008; 36:367-8.

8. Abbas MA, Collins JM, Olden KW, Kelly KA. Spontaneous intramural small bowel hematoma: clinical presentation and longterm outcome. Arch Surg 2002;137:306-10.

9. Sorbello MP, Utiyama EM, Parreira JG, et al. Spontaneous intramural small bowel hematoma induced by anticoagulant therapy: review and case report. Clinics (Sao Paulo) 2007;62:785-90.

10. Preston FE, Laidlaw ST, Sampson B, Kitchen S. Rapid reversal of oral anticoagulation with warfarin by a prothrombin complex concentrate (Beriplex): efficacy and safety in 42 patients. Br J Haematol 2002;116:619-24.

11. Proceedings of the Seventh ACCP Conference on Antithrombotic and Thrombolytic Therapy: evidence-based guidelines. Chest 2004;126:172S-173S.

12. Van Walraven C, Hart RG, Singer DE, et al. Oral anticoagulants versus aspirin in nonvalvular atrail fibrillation. JAMA 2002;288: 2441-8. 